\title{
La recherche au subjectif imparfait
}

Introduction au numéro 94 de la revue Communications paru en 2014 sous le titre Chercher. S'engager?

\section{Pierre Alphandéry, Sophie Bobbé}

Plusieurs raisons nous ont incité à interroger les pratiques de recherche en partant de la subjectivité des auteurs et du contexte de production de leurs travaux. Jamais sans doute, la société française n'a été à ce point l'objet d'investigations mais la multiplication des formes de spécialisation ou de professionnalisation, et la difficulté des sociologues et des anthropologues à dégager des bases communes disciplinaires rendent de plus en plus problématique la critique et l'usage des connaissances produites. Cette situation a aussi donné lieu à des initiatives importantes épousant les champs disciplinaires, qu'il s'agisse d'évaluer la possibilité d'une théorie sociologique générale ${ }^{1}$. Ou encore qu'il s'agisse en matière ethnographique du travail de synthèse encyclopédique réalisé par Daniel Céfaï ${ }^{2}$. Notre objectif est ici différent: (2) nous nous sommes placés délibérément du point de vue de la subjectivité des chercheurs qui éclairent, à partir de leur histoire, certains des ressorts de leurs activités professionnelles. Cette démarche nous a semblé d'autant plus nécessaire que depuis trois décennies environ, s'est produite une profonde inflexion des pratiques, des valeurs et des questions (issues de dynamiques internes ou externes) tant en sociologie qu'en ethnologie. Durant ce laps de temps, les sciences humaines et sociales ont évolué au diapason du reste de la société en se laissant gagner par des formes massives de précarité statutaire, une organisation marquée par la course à la production, la concurrence et la compétition, une gestion managériale fondée sur la communication et des mécanismes d'évaluation permanente.

On peut d'ailleurs se demander si les connaissances produites par les sciences humaines et sociales n'ont pas contribué à ce mouvement, en s'attachant à mettre au jour la complexité des logiques d'acteurs ou encore les mécanismes de fragmentation sociale ${ }^{3}$. Les pratiques de recherche ont participé à faire basculer les stratégies de contestation vers des logiques davantage ancrées sur la protection, l'écoute et la gestion pragmatique. De plus en plus procédurales, les politiques publiques, de leur côté, s'attachent à mettre en place des formes locales de délibération où des points de vue se confrontent. De nombreux domaines de l'action publique (en particulier ce qui relève de l'environnement) font appel au savoir faire et aux connaissances de chercheurs en sciences sociales de plus en plus professionnalisés qui, se plaçant en position d'expertise, produisent diagnostics et évaluations. L'importance prise par ces recherches menées « au plus près des acteurs » n'est pas nouvelle pour les ethnologues dont l'objet est précisément le discours de l'Autre. En revanche, elle a amené nombre de sociologues à pratiquer l'observation participante, à s'intéresser aux connaissances des acteurs locaux et à diverses techniques de recueil d'informations sur le terrain. Ce faisant, cette approche qui se défie des théories trop larges présente le risque de conduire à une standardisation des recherches qui, en devenant opératoires, les allègent de leur portée critique. 
Pour toutes ces raisons, il nous a paru important de (3) solliciter plusieurs chercheurs à propos de leur posture et de leurs trajectoires personnelle et professionnelle. Nous entendons par le terme de «posture » la position que le chercheur occupe par rapport à ses objets de recherche, à ses interlocuteurs, à son terrain, et aussi à ses pairs et aux institutions qui structurent son activité. Loin d'être immuable, cette posture s'inscrit dans un rapport à la recherche qui ne cesse d'évoluer dans un mouvement que nous avons qualifié de cheminement. On rentre dans le monde de la recherche avec des attaches biographiques, géographiques, des appartenances sociales et culturelles qui vont peser sur le processus qui constituera le chercheur en sujet autonome inscrit dans un contexte et une histoire. La posture du chercheur renvoie donc à un ensemble particulièrement massif d'éléments d'ordres matériels, subjectifs et structurels ${ }^{4}$ par lesquels il se trouve immergé et engagé dans le social.

Il n'est pas besoin d'insister sur les rapports étroits que les sciences sociales ont entretenus avec les événements qui ont modelé la société française et son État. Pour n'en rester qu'à l'après-guerre, période principalement abordée dans les articles, l'émergence de l'ethnologie et de la sociologie apparaît profondément liée à la colonisation et à la recherche de modernisation. La sociologie en est ressortie fortement divisée aussi bien sur un plan épistémologique que sur son rôle dans la société. D'une part, les explications de type utilitariste ont gagné en influence, portées par le modèle de l'homo economicus ; d'autre part, le rôle de la sociologie donne lieu à des oppositions structurées autour de deux groupes. Le premier rassemble ceux qui se considèrent avant tout comme experts du social, se réclamant d'un savoir faire constitué souvent dans un domaine spécialisé. Le second groupe réunit ceux qui se réclament d'un point de vue critique, ancré dans une conception de la production de la société qui met en avant les classes sociales, la domination, le rôle de la culture, etc. On assiste, durant les années 1970, à un essor important de la commande publique qui va multiplier les recherches contractuelles aussi bien dans le domaine de la sociologie que dans celui de l'ethnologie qui commence à investir le «proche». Cette institutionnalisation des sciences sociales ne supprime nullement les différences de postures. En sociologie comme en ethnologie, les courants se sont durcis ce qui n'a guère incité la grande masse des « hors statuts » recrutés au début des années 1980 à assumer leur biographie et leurs diverses expériences du monde.

(1) La pluralité des attaches, leurs liens avec le choix des objets de recherche, des méthodes et des questions ont longtemps été passés sous silence par les chercheurs euxmêmes comme si ces thèmes risquaient de discréditer la démarche scientifique. Or, la diversité et la fécondité de la recherche tiennent à la pluralité des postures et des cheminements dont on trouvera l'illustration dans les contributions ici rassemblées.

Dans un texte célèbre, Max Weber questionnait déjà la «signification » du travail scientifique qui, une fois achevé, «n'a d'autre sens que de faire naître de nouvelles questions. ${ }^{5}$ » Pourquoi, poursuivait-il, se livrer à une occupation qui n'a pas de fin ? Certes le savant participe ainsi à « l'intellectualisation» du monde et à sa rationalisation et donc au progrès, mais son travail a aussi une portée éducative qui peut contribuer à responsabiliser les hommes : «Si nous sommes en tant que savants à la hauteur de notre 
tâche (...), nous pouvons alors obliger l'individu à se rendre compte du sens ultime de ses propres actes, ou du moins l'y aider. ${ }^{6} \gg$ (4)Serait-il possible d'imaginer qu'un chercheur choisisse de traiter d'un sujet indépendamment de sa biographie, de ses préoccupations personnelles et des enjeux de l'époque ? Nous partirons du point de vue que le choix d'objets et/ou l'approche retenue relève(nt) à la fois de la «grande » et de la «petite » histoire. Dans ce qui suit, la grande histoire sera incarnée par le politique. La petite histoire se nourrissant de la subjectivité et des formes d'engagement de chacun.

Le rôle instituant du politique

L'âge et l'appartenance générationnelle d'une grande partie des contributeurs de ce numéro expliquent combien la politique et le politique ont joué et/jouent un rôle important dans les choix déterminant leur posture. Précisons cependant que cette assertion n'est pas vraie pour tous les auteurs. De plus, pour ceux qui sont concernés, le sens implicite donné à la notion de politique n'est pas toujours identique. Il nous a paru utile de nous adosser à la distinction que Chantal Mouffe introduit entre le politique et la politique. Celle-ci remarque que la caractéristique de la démocratie pluraliste comme forme spécifique d'ordre politique est d'instaurer une distinction entre les catégories d'ennemi et d'adversaire. Ainsi l'opposant n'est pas considéré comme un ennemi à abattre mais comme un adversaire dont la présence est légitime et qui doit être toléré. L'ennemi ne disparaît pas pour autant; il désigne ceux qui mettent en question les bases même de l'ordre démocratique. Chantal Mouffe propose ainsi « de distinguer le politique qui est lié à la dimension d'antagonisme et d'hostilité qui existe dans les rapports humains... et la politique qui vise à établir un ordre, à organiser la coexistence humaine dans des conditions qui sont toujours conflictuelles car traversées par le politique. ${ }^{7}$ »

Le politique ainsi défini apparaît bien présent dans un certain nombre des articles que nous avons réunis, structurant des visions du monde, hiérarchisant les priorités et nécessitant une traduction dans l'action contre des ennemis incarnant des formes de domination radicales et insupportables (la lutte contre le colonialisme, les réflexions autour de la Shoah et le nazisme, les guerres d'Algérie et du Vietnam, le soutien aux travailleurs sans papiers...). Cette présence du politique particulièrement forte après 1968, jusqu'au début des années 1980, n'influence pas tous les auteurs «politisés » de la même manière. Sophie Wahnich choisit de travailler sur la figure de l'étranger depuis la révolution française en posant la question suivante : «qu'est-ce qui se joue dans le contrôle social où l'on demande à l'étranger de faire la preuve de son patriotisme et ainsi de son innocence?» Pour sa part, Emmanuel Terray, citant Carl Schmitt, tient à distinguer l'action politique qui sépare les amis des ennemis, de la recherche où l'on doit nouer des rapports avec toutes les personnes susceptibles d'apporter des informations importantes. A contrario, Alban Bensa pourtant moins imprégné de culture politique lorsqu'il arrive en Nouvelle-Calédonie, ne conçoit pas ses travaux sur les communautés Kanak comme séparés de la lutte contre la spoliation et le racisme dont celles-ci sont victimes. La posture de Michel Dreyfus apparaît intermédiaire. Historien, se réclamant des valeurs de la gauche laïque, il a longuement travaillé sur le mouvement ouvrier sans toutefois aborder un sujet «brûlant» largement délaissé par son «camp » politique, l'antisémitisme issu de la gauche. Il raconte le chemin parcouru et les obstacles rencontrés dans sa recherche la plus difficile, aussi bien sur le plan politique que personnel. 
Dans sa seconde catégorie complémentaire de la première, Chantal Mouffe appréhende la politique comme englobant ce qui concerne le vivre ensemble, en particulier à travers la constitution d'espaces publics, ces lieux où s'opèrent la confrontation des idées et la mise en commun des problèmes. Cette approche est nourrie de l'héritage issu des écrits de Hannah Arendt, Claude Lefort, Jürgen Habermas ou Cornélius Castoriadis, revendiqué par des chercheurs qui assument une posture normative, s'éloignant ainsi de la neutralité axiologique chère à Max Weber. Geneviève Decrop et Yves Dupont rappellent l'importance de la dynamique de débats qui a marqué la décennie d'après 68 dans leur formation intellectuelle. La première souhaitant aux jeunes de connaître aussi un même «bonheur public», le second évoquant son peu d'attirance pour le dogmatisme « gauchiste » et sa chance d'avoir bénéficié d'une effervescence culturelle lui ayant permis de découvrir l'anthropologie économique et des auteurs tels que Pierre Clastres, Marcel Mauss, Karl Polanyi, etc. De son côté, Sergio Dalla Bernardina décrit l'ambiance saturée de politique des années 1970 en Italie où l'ethnologie était conçue comme une forme de « réhabilitation de la culture populaire ». Partie prenante de cette aspiration à faire cause commune avec l'Autre qu'il qualifie «d'enthousiasme post gramscien», il évoque néanmoins le malaise qu'il éprouvait à endosser cette posture, malaise qui devait le conduire à transformer sa conception de l'enquête ethnographique en questionnant l'empathie comme norme.

En revanche, l'importance des enjeux que représentent la constitution d'espaces de débats réellement pluralistes a conduit Yves Dupont et Geneviève Decrop à travailler, voire à soutenir des acteurs impliqués dans la remise en cause du productivisme, des OGM ou de certaines institutions. De la même manière, tout en préservant de la tentation de devenir expert, Alain Bertho souhaite initier avec les gens d'abord et les acteurs institutionnels ensuite des « dispositifs d'identification de problèmes et de formulations de solutions. » Cette volonté de travailler avec des acteurs marginalisés par le «système » ne s'exprime pas de la même manière aujourd'hui, tant diffèrent les contextes national et international. Elle pose aussi la question du rôle des institutions dans différents domaines. Selon Geneviève Decrop, qui a choisi de « rester à l'extérieur », les institutions protègent tout autant qu'elles étouffent. On ajoutera que leur rôle n'a cessé de se renforcer en matière de gestion de la recherche et qu'elles constituent des cadres cognitifs et normatifs au sein desquels la transmission des expériences, d'une génération à l'autre, s'avère fort difficile.

La troisième forme de présence du politique est plus difficile à identifier que les deux premières, car elle est constituée par un ensemble d'éléments dont le rapprochement peut sembler paradoxal. Les grandes catégories collectives fondatrices de la souveraineté et de l'exercice du pouvoir (la nation, la société, l'État, le peuple) se fragmentent au profit de lieux de pouvoir diversifiés (collectifs divers, entreprises, collectivités locales, science) caractérisés par des formes d'action, des réseaux, des procédures, des normes, etc. Les couples de catégories fondatrices de notre organisation collective (privé/public, individu/collectif, local/global, sauvage/domestique, etc.) voient leurs frontières redéfinies. On s'intéresse alors aux instruments de gouvernement, aux dispositifs ${ }^{8}$, aux réseaux, en bref aux divers moyens qui organisent l'action publique. Cette troisième figure du politique suscite une série de questions. Elles concernent notamment le rôle des 
connaissances et leur rapport au pouvoir. Elles portent enfin sur les conséquences qu'engendre la scientifisation du politique : celui-ci ne risque-t-il pas de se réduire progressivement à la gestion du vivant ? Ainsi Geneviève Decrop, évoquant les scènes du risque, se demande si la position de facilitateur de communication entre les acteurs qu'occupent les sociologues ne les conduit pas à se transformer en experts au service de leurs commanditaires. Sa réponse reste nuancée, mais cette question la conduit à choisir de travailler avec des associations de victimes, créant un espace du politique à travers la mise en cause de puissantes institutions.

Cette grille de lecture n'épuise pas la question politique, mais suggère l'importance des valeurs et des aspirations collectives, des crises sociales et des configurations historiques dans la construction des postures et des trajectoires des contributeurs. On notera toutefois qu'une partie des contributions peut parfaitement ignorer le rôle instituant du politique, telles celles d'Isabelle Mauz et de Sophie Bobbé qui ancrent ailleurs leurs questions et les formes de leur engagement. Quoi qu'il en soit, ce qui relève de ce qu'on peut qualifier de « grande histoire » ne trouve à s'exprimer qu'en fonction d'une « petite histoire ».

\section{La subjectivité}

En 1922, alors qu'il rédigeait Les Argonautes du Pacifique occidental, Bronislaw Malinowski évoquait déjà le rôle de l'équation personnelle ${ }^{10}$ dans le travail d'enquête. De façon plus ou moins explicite suivant les époques et les courants de pensée, la question de l'irruption $\mathrm{du}$ «je » a très tôt traversé la discipline ethnologique, que ce soit pour la critiquer, la revendiquer ou encore l'utiliser pour expliciter la différence de statut du journal de terrain et du travail académique, et le traitement éditorial distinct qui leur sont réservés ${ }^{11}$. Comme le note Jeanne Favret-Saada, «... L'ethnographie a légitimement constitué ses procédures de recherche et ses critères de validité autour de la notion d'objectivation. C'est bien en parlant de l'indigène comme d'un objet, comme d'un “autre", en le désignant comme sujet de l'énoncé ("ill" pratique ou dit ceci ou cela) qu'est fondée la possibilité d'un discours sur une culture différente, sur un objet qui ne sera pas moi. Encore faut-il pourtant, si l'on veut que ce discours soit plausible ou même intelligible, que ce moi s'énonce lui-même et dise à l'adresse de qui il tient ce discours sur l'autre : car seul un être humain se dénommant lui-même "je" peut en désigner un autre comme "il" ; et il ne saurait le faire qu'à l'adresse d'un " $t u$ ". ${ }^{12}{ }$ "En ethnologie comme en sociologie, la recherche se déploie dans l'intersubjectivité. Par sa présence, son comportement, ses questionnements, le chercheur entre en relation avec son terrain, autrement dit avec des « ils », et l(es)'affecte(nt) tout comme il est lui-même affecté.

L'expression de la subjectivité du chercheur se décline de façons diverses dans les contributions de ce numéro. Certains auteurs ont choisi de l'évoquer à partir de la présentation du cadre théorique et des textes de référence qui les nourrissent.

Pour d'autres, la subjectivité se donne à voir dans la mise en scène de soi (le « je » chercheur) et dans les modes d'écriture (le «je»auteur) notamment à travers 
l'explicitation des choix tant méthodologiques qu'épistémologiques - c'est précisément ce qu'expérimentent Alban Bensa, Sophie Bobbé, Sergio Dalla Bernardina, Geneviève Decrop et Isabelle Mauz lorsqu'ils questionnent leurs façons de faire leur métier de chercheur, leur rapport au terrain et à leurs informateurs. La subjectivité n'induit pas systématiquement un rapport d'empathie. Dans leurs contributions respectives, Sergio Dalla Bernardina et Isabelle Mauz interrogent le rapport d'empathie que le chercheur entretient avec ses informateurs. Le premier tend à considérer l'empathie comme une illusion ou un piège dans la relation d'enquête : ainsi, le chercheur ne trahirait-il pas ses informateurs en leur faisant croire à une possible convergence des représentations «indigènes» et en dissimulant des sentiments peu honorables (le narcissisme, le paternalisme ou le populisme) derrière une empathie de façade ? En contrepoint, le travail d'Isabelle Mauz relate une expérience d'une sociologie participative au cours de laquelle elle invite ses informateurs à sa table en les traitant comme des pairs.

Une troisième forme d'énonciation de la subjectivité tient à l'intrication, l'entrelacement de l'histoire biographique du chercheur et du choix de ses thématiques investies, comme l'illustrent les contributions de Michel Dreyfus et d'Yves Dupont. Ce faisant, la prise en compte de leur subjectivité «... est peut-être moins un obstacle à vaincre qu'une ressource à utiliser dans la production des connaissances. ${ }^{13}$ » Autre forme d'entrelacement entre l'histoire biographique du chercheur et sa posture scientifique est celle de Sophie Wahnich défendant la prise en compte de sa subjectivité mise au service d'une quête de vérité.

En définitive, la subjectivité apparaît comme la condition sine qua non à toute forme d'engagement sans pour autant l'appeler.

\section{L'engagement}

Depuis Max Weber et Norbert Élias ${ }^{14}$, par-delà les disciplines de référence et les traditions de pensée, de nombreux chercheurs se sont interrogés sur leur pratique au regard des rapports entre distanciation et implication, neutralité et engagement, certains allant jusqu'à postuler une possible neutralité engagée ${ }^{15}$. Il n'est pas ici question de régler cette épineuse question ni de la dissimuler sous le boisseau mais bien de replacer le chercheur en tant que sujet au cœur de sa recherche en mettant au jour les choix et les sensibilités qui ont présidé à son travail, ce qui justifie sur le plan rhétorique l'emploi du « je » dans ses articles. Qu'elle soit abordée frontalement ou de façon périphérique, la question de l'engagement est au cœur de toutes les contributions, mettant en question la neutralité axiologique souvent érigée en norme ou assimilée, voire justifiée par les procédures d'objectivation.

Polymorphe, l'engagement varie selon la trajectoire biographique du chercheur, infléchissant son rapport au terrain de recherche (à ses interlocuteurs), à son champ disciplinaire comme à sa problématique. Associé par certains à la lutte politique, notamment lorsqu'il s'applique à la défense de causes sur la scène publique, il peut être volontairement circonscrit à la vie citoyenne du chercheur (Emmanuel Terray) ou par 
capillarité, affecter sa recherche. Dans le cas d'Alain Bertho, Geneviève Decrop et Yves Dupont, l'engagement se poursuit dans la pratique scientifique. A contrario, il peut aussi découler de l'interpellation du terrain (Alban Bensa). Animant différemment le chercheur et ses questionnements scientifiques, l'engagement le conduit parfois à assumer une réduction de la distance avec ses interlocuteurs (sans pour autant se retrouver à leur service) pour prendre publiquement position à leur côté (Alban Bensa, Alain Bertho, Geneviève Decrop, Yves Dupont), notamment lorsque ces derniers ne sont absents sur la scène publique. Ces choix apportent des réponses différentes à la question des rapports entre l'engagement citoyen dans la vie publique et la pratique professionnelle. Ils exposent leurs auteurs à assumer un point de vue situé qui les conduit parfois à réinterroger le regard majoritairement partagé par leur communauté disciplinaire comme l'illustre les positions épistémologiques défendues par Sophie Wahnich lorsqu'elle affirme vouloir assumer une histoire qui prend tout son sens dans l'interrogation du présent : ainsi « le rapport passé/présent si l'on veut prendre au sérieux le rapport au présent suppose un historien situé, voire engagé ».

En écho à l' «ego-sociologie critique » d'Yves Dupont, l' «ego-histoire critique » de l'historien Michel Dreyfus réintroduit le sujet au centre de sa recherche, notamment lorsqu'il déplie les liens intimes de sa propre histoire et la façon dont il s'est saisi de son dernier thème de recherche. Si l'on connaît les risques inhérents à cette approche ${ }^{16}$, on peut également lui reconnaître une vertu, celle de stimuler la réflexivité du chercheur, occasion pour chacun de mettre au jour sa cuisine personnelle, son bricolage intellectuel et, in fine sa posture scientifique. Yves Dupont se prête à ce mécano en évoquant l'habitus qui désigne, on le sait, l'influence que le milieu d'origine d'une personne exercera sur elle toute sa vie durant, ce qui permet à cet auteur de compléter la définition initiale théorisée par Bourdieu en rappelant que l'habitus peut également constituer le socle à partir duquel cette personne choisira l'usage qu'elle en fait, exerçant alors sa liberté en situation.

L'engagement trouve également à s'exprimer sur le plan de l'éthique professionnelle. Questionnant la place et le rôle de ses informateurs, Isabelle Mauz met en cause la règle sociologique selon laquelle l'interlocuteur est cantonné dans son rôle d'informateur. En tentant de rééquilibrer la relation d'enquête et de gommer les rapports de pouvoir qui lui sont inhérents, elle choisit de considérer ses informateurs comme ses pairs. Ce faisant, elle prend le parti de les inviter à discuter ses propres résultats, prônant ainsi une sociologie plus participative, qui témoigne d'une forme déontologique de son engagement professionnel.

Qu'il soit au bénéfice d'un tiers - l'institution de tutelle (Isabelle Mauz), le politique (Yves Dupont, Sophie Wahnich), les acteurs (Alban Bensa, Alain Bertho, Geneviève Decrop) ou encore du sens et de la transmission (Sergio Dalla Bernardina,) l'engagement ici trouve toute son expression. 
${ }^{1}$ Revue du Mauss, $\mathrm{n}^{\circ}$ 24, « Une théorie sociologique générale est-elle pensable ? », 2004.

${ }^{2}$ Par exemple Daniel Céfaï, L'engagement ethnographique, Paris, EHESS, 2010.

${ }^{3}$ Danilo Martuccelli, «Sociologie et posture critique » in B. Lahire (dir.), À quoi sert la sociologie ?, Paris, La Découverte, 2002, p. 147.

${ }^{4}$ Claude Dubar, «Les tentatives de professionnalisation des études de sociologie : un bilan prospectif », in Bernard Lahire (dir.), À quoi sert la sociologie ?, Paris, La Découverte, 2002, p. 105.

${ }^{5}$ Max Weber, Le savant et le politique (1919), Paris, collection 10/18, 1991, p. 87.

${ }^{6}$ Max Weber, Ibidem, 1991, p. 115.

${ }^{7}$ Chantal Mouffe, Le politique et ses enjeux, Paris, La Découverte, 1994, p. 10-11.

${ }^{8}$ Dispositif est ici entendu comme un réseau d'objets hétérogènes en interdépendance, comportant des humains et des non humains, des éléments matériels et discursifs. Cf. Valérie Boussard, Salvatore Maugeri (dir.), Du politique dans les organisations, Paris, L'Harmattan, 2006, p. 3.

${ }^{9}$ Pierre Birnbaum soulignait déjà en 1975 la montée de l'empirisme contre la politique et l'idéologie dans les sciences politiques anglo-saxonnes, traduction de la vision dominante du politique comme «administration scientifique des choses » par une élite spécialisée tenant la société à l'écart des affrontements. Cf. La fin du politique, Paris, Le Seuil, 1975.

${ }^{10}$ L'expression « équation personnelle » est ici entendue comme « l'ensemble des variables perceptives et interprétatives composant le chiffre individuel de chaque chercheur. » Cf. Sergio Dalla Bernardina, " "Équation personnelle" et statut de l'observateur dans la tradition ethnologique », Sociologie du Sud-Est. Revue de sciences sociales, $\mathrm{n}^{\circ}$ 59-62, 1989, $\mathrm{p}$. 8.

${ }^{11}$ Voir à ce sujet, Sergio Dalla Bernardina, « "JE” interdits. Le regard presbyte de l'ethnologue » (2000), in Georges Ravis-Giordani (dir.), Ethnologie(s). Nouveaux contextes, nouveaux objets, nouvelles approches, Paris, CTHS, 2009 , p. 18-40.

${ }^{12}$ Souligné en italiques par Jeanne Favret-Saada, Les mots la mort, les sorts. La sorcellerie dans le Bocage, Paris, Gallimard, 1977, p. 42-43.

${ }^{13}$ Jean Poupart, «Discours et débats autour de la scientificité des entretiens de recherche », Sociologie et sociétés, vol XXV, n 2, 1993, p. 108.

${ }^{14}$ Engagement et distanciation, Paris, Fayard, 1983.

${ }^{15}$ Nathalie Heinich, « Pour une neutralité engagée », Questions de communication, $\mathrm{n}^{\circ} 2,2002$.

${ }^{16}$ Jean-Pierre Olivier de Sardan, « Jeu de la croyance et "je" ethnologique : exotisme religieux et ethno-égo-centrisme », Cahier d'études africaines, vol 28, n 111-112, 1988, p. 527-540. 